\section{A) Check for updates}

Cite this: Food Funct., 2022, 13, 2415

\title{
Biological properties and therapeutic applications of garlic and its components
}

\author{
Lucía Melguizo-Rodríguez, a,b Enrique García-Recio, ${ }^{\text {b,c }}$ Concepción Ruiz, (DD a,b,d \\ Elvira De Luna-Bertos, ${ }^{a, b}$ Rebeca Illescas-Montes (iD *a,b and Víctor J. Costela-Ruiz ${ }^{b, e}$
}

\begin{abstract}
Garlic is one of the most widely employed condiments in cooking. It has also been used since ancient times in traditional plant-based medicine, largely based on its organosulfur compounds. The objective of this study was to provide updated information on the biological and therapeutic garlic properties. Garlic has been found to possess important biological properties with high therapeutic potential, which is influenced by the mode of its utilization, preparation, and extraction. It has been attributed with antioxidant, anti-inflammatory, and immunomodulatory capacities. Garlic, in particular its organosulfur compounds, can maintain immune system homeostasis through positive effects on immune cells, especially by regulating cytokine proliferation and expression. This may underlie their usefulness in the treatment of infectious and tumor processes. These compounds can also offer vascular benefits by regulating lipid metabolism or by exerting antihypertensive and antiaggregant effects. However, further clinical trials are warranted to confirm the therapeutic potential of garlic and its derivatives.
\end{abstract}

Received 22nd September 2021 Accepted 11th February 2022

DOI: $10.1039 / \mathrm{d} 1 \mathrm{fo} 03180 \mathrm{e}$

rsc.li/food-function isolation of the organosulfur components of garlic is challenging due to their physicochemical properties, and variability in their proportions among garlic preparations hampers reproduction and validation of the effects observed in different studies. ${ }^{6}$ Also, despite the fact that garlic supplementation has been classified as a safe product, some non-serious gastrointestinal side effects have been reported. ${ }^{9,10}$

The aim of this study was to provide an update of scientific evidence on the biological properties of garlic and its chemical components and their relevance for the management of health and disease.

\section{Characteristics and composition of garlic}

Garlic contains more than 200 chemical compounds with multiple properties. It is $65 \%$ water, $28 \%$ carbohydrates, $2.3 \%$ organosulfur compounds, $2 \%$ proteins, $1.2 \%$ free amino-acids, and $1.5 \%$ fiber. It also contains fat-soluble vitamins (vitamin $\mathrm{A}$, vitamin $\mathrm{K}$, and vitamin $\mathrm{E}$ ), water-soluble vitamins (vitamin C, B-complex vitamins: B1, B2, B3, B6, and B8), and minerals ( $\mathrm{Ca}, \mathrm{Fe}, \mathrm{Mg}, \mathrm{P}, \mathrm{K}, \mathrm{Na}$, and $\mathrm{Zn}$ ). Organosulfur compounds give garlic its characteristic taste and odor as well as its pharmacological properties. ${ }^{11}$

Moreover, garlic has both non-volatile and volatile organosulfur compounds. Non-volatile precursor compounds of sulfur in garlic include $\gamma$-glutamyl-S-allyl---cysteine and its sulfoxide, known as alliin. Alliin is only present in intact garlic, being transformed into other compounds when the bulb is macerated or crushed. In contrast, volatile organosulfur com- 
pounds are generated during the processing of garlic products, being divided into three subgroups according to their chemical nature (Fig. 1): (a) thiosulfinates, which result from the conversion of sulfoxides by enzymatic reaction when raw garlic is processed. (b) Volatile organosulfurs such as allicin (diallyl thiosulfinate), one of the most biologically active compounds, which is absent in intact garlic but produced by the reaction of alliin with the enzyme alliinase when the bulb is ground, crushed, or damaged. Allicin is highly unstable and instantly decomposes into diallyl sulfide (DAS), diallyl disulfide (DADS), diallyl trisulfide (DATS), and/or sulfur dioxide $\left(\mathrm{SO}_{2}\right)$, among other compounds. This subgroup also includes methyl allyl disulfide and methyl allyl trisulfide. (c) Water-soluble organosulfur compounds, generated during the aqueous or alcoholic extraction of garlic through the decomposition of $\gamma$-glutamyl-Sallyl---cysteine into $S$-allyl-L-cysteine (SAC).

In summary, intact bulbs contain SAC and $\gamma$-glutamyl- $S$ allyl-L-cysteine sulfoxide, vapor-distilled oils contain volatile sulfide compounds (DADS, DATS, DAS), dry garlic powder is rich in alliin and DADS, and garlic macerates are rich in organosulfur compounds, ditiins, and E,Z-ajoenes. Finally, mashed and ethanol-soaked aged garlic extract (AGE) contains SAC and $S$-allyl-mercaptocysteine (SAMC) and has shown more biological properties in comparison to the aforementioned preparations. ${ }^{12-15}$ Multiple factors influence the bioactivity of garlic components, such as extraction, preparation, temperature or storage. In this context, it is reported that longer cooking time and increased temperature may reduce the proportion of phenolic compounds, such as hesperetin and 3/ 4-hydroxybenzoic acid, and flavonoid. ${ }^{16,17}$ It should be noted that absorption, metabolism and excretion are the main indices of bioavailability of an ingested compound. Allicin and allicin-derived compounds are rapidly metabolized to allyl methyl sulfide, which is the main metabolite of allicin and an active metabolite (Lawson and Wang, 2001). ${ }^{18}$ However, information on the bioavailibity of organosulfur compounds is

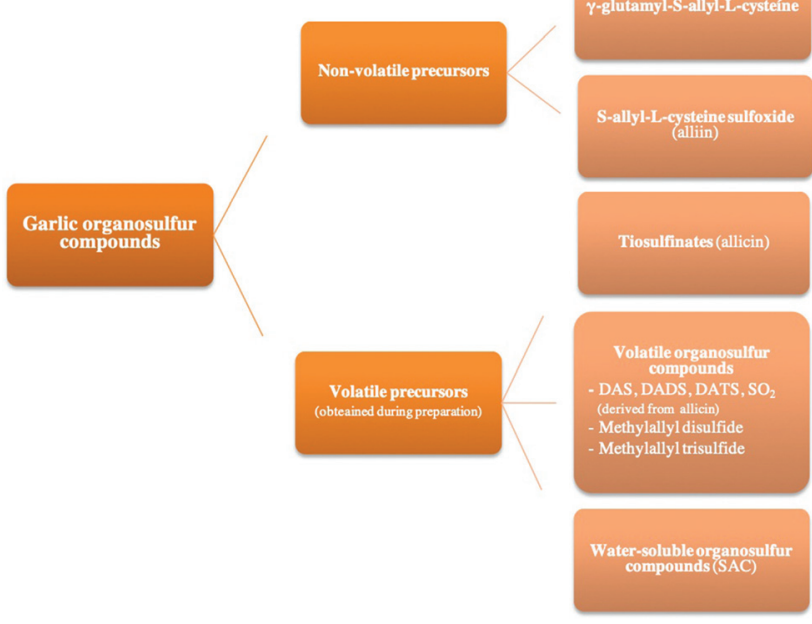

Fig. 1 Organosulfur compounds of garlic. limited. Thus, Miękus et al. ${ }^{19}$ suggested the need to conduct a series of clinical trials in a significant number of volunteers to optimize doses and understand the factors that determine bioavailability.

\section{Biological activity of garlic}

The different biological mechanisms of garlic are summarized in Table 1.

\section{Antioxidant activity}

The oxidation of DNA, proteins, and lipids by reactive oxygen species (ROS) plays a key role in aging and in the development of cancers and cardiovascular, inflammatory, neurodegenerative, and age-related diseases. ${ }^{20,21}$

The antioxidant potential of garlic largely derives from its saponins, flavonoids, organosulfur compounds, and phenolic compounds. This potential is higher in aqueous extracts (e.g., AGE) than in fresh garlic or other preparations, likely due to the presence of water-soluble organosulfur compounds with antioxidant capacity (e.g., SAC and SAMC). Furthermore, a synergetic antioxidant effect has been observed between these compounds and flavonoids, saponins, and certain micro- and macro-nutrients also present in aqueous garlic extracts. ${ }^{11,14}$ Phytochemicals in these extracts can exert a double action by favoring the expression of antioxidant enzymes (e.g., superoxide dismutase [SOD] and catalase) and increasing cell glutathione (GSH) levels. ${ }^{22,23}$ In a recent meta-analysis of randomized controlled trials, serum SOD levels and total antioxidant capacity were found to be increased by supplementation with $80-4000 \mathrm{mg}$ day $^{-1}$ of garlic for $2-24$ weeks, with a reduction in malondialdehyde production. ${ }^{24}$ An in vitro study found that ajoene, a chemically stable by product of garlic, can activate nuclear factor 2 (Nrf2), regulating the expression of glutamatecysteine ligase (GCL), raising levels of GSH, a potent antioxidant, and increasing the expression of other genes that encode cysteine-metabolizing enzymes. ${ }^{25} \mathrm{Nrf} 2$ controls the expression of numerous genes involved in the defense against xenobiotic agents and oxidative stress and is considered a key regulator of oxidative stress and the inflammatory response. For instance, it induces the gene expression of heme oxygenase-1 (HO-1), an enzyme that catalyzes degradation of the hemo group, generating carbon monoxide (CO), biliverdin, and iron. This enzyme is activated by oxidative stress or by the presence of cytokines or other mediators during inflammatory processes. ${ }^{25}$ In a study of human endothelial cells, AGE was found to reduce oxidative stress in a dose-dependent manner by activating the Nrf2 pathway through an increase in the expression of GCL and HO- $1 .{ }^{26}$ Oral administration of AGE (100-200 mg kg-1) to male albino rats with indomethacininduced gastric inflammation had a gastroprotective effect that was attributed to its anti-inflammatory and antioxidant properties. ${ }^{27}$ In this regard, it was reported in an in vitro study that allicin attenuates oxidative stress responses induced by lipopolysaccharides (LPS) of Gram-negative bacteria via the 
Table 1 Key findings of the biological activity of garlic

\begin{tabular}{|c|c|}
\hline Biological acivity & Mechanism of action \\
\hline \multirow[t]{3}{*}{ Antioxidant activity } & $\begin{array}{l}\text { Garlic phytochemicals could promote the expression of antioxidant enzymes, such as } \\
\text { SOD or catalase, and increase cellular GSH levels. } \\
\text { Supplementation with } 80-4000 \mathrm{mg} \mathrm{day}^{-1} \text { of garlic for } 2-24 \text { weeks increases serum SOD } \\
\text { levels, increasing total antioxidant capacity through a reduction of malondialdehyde. }\end{array}$ \\
\hline & $\begin{array}{l}\text { Ajoene exerts its antioxidant function through the activation of Nrf2, which regulates } \\
\text { GCL expression and increases GSH levels, among other mechanisms of action. Likewise, } \\
\text { AGEs could reduce the oxidative stress of endothelial cells by activating the Nrf } 2 \text { pathway, } \\
\text { through an increase in the expression of GCL and HO-1. } \\
\text { Allicin attenuates oxidative stress responses caused by different factors through the Nrf2 } \\
\text { pathway and regulation of apoptosis. }\end{array}$ \\
\hline & $\begin{array}{l}\text { AGEs play an antioxidant role against damage to DNA structures caused by ultraviolet } \\
\text { radiation, performing antimutagenic, anticarcinogenic and reparative activities on } \\
\text { damaged structures. }\end{array}$ \\
\hline \multirow[t]{5}{*}{$\begin{array}{l}\text { Anti-inflammatory } \\
\text { activity }\end{array}$} & $\begin{array}{l}\text { A reduction in adipocyte IL- } 6 \text { and MCP-1 secretion, as well as up-regulation of the } \\
\text { expression of genes involved in inflammatory response has been documented after alliin } \\
\text { treatment. }\end{array}$ \\
\hline & $\begin{array}{l}\text { Treatment with garlic extracts on mononuclear cells showed inhibition of IL-17 secretion. } \\
\text { IL-14 secretion was not affected after treatment with garlic extract on mononuclear cells. }\end{array}$ \\
\hline & AGE reduces the production of IL-12, TNF- $\alpha$ and IL- 6. \\
\hline & $\begin{array}{l}\text { Garlic and its components may modulate the inflammatory response induced by } \mathrm{T} \\
\text { helper cells. }\end{array}$ \\
\hline & $\begin{array}{l}\text { Garlic components have the potential to inhibit NF- } \kappa \text { B, thereby reducing the } \\
\text { inflammatory response mediated by TNF- } \alpha \text {, IL-1, IL- } 6, \text { MCP- } 1 \text { and IL-12, among other }\end{array}$ \\
\hline
\end{tabular}

Antimicrobial activity Garlic extracts have been shown to have antibiofilm and antibacterial effects against Bacillus cereus, Staphylococcus aureus, Micrococcus spp., Escherichia coli, Klebsiella spp., Proteus spp., Aspergillus versicolor, Penicillum citrinum, and Penicillium expansum.

Anti-fungal activity of garlic against Penicillium funiculosum and Candida albicans has been demonstrated.

Garlic has been shown to have antiviral activity through inhibition of retrotranscription and viral DNA synthesis capacity.

Garlic and its organosulphur and flavonoid components exert antiviral action against SARS-CoV-2 infection via inhibition of the 6LU7 protein of the active region of the pathogen.

Garlic has therapeutic action in COVID-19 disease by strengthening immune system cells and decreasing the secretion of proinflammatory cytokine and leptin.

Garlic extract inhibits the penetration and proliferation of the influenza A (H1N1) virus. Ajoene obtained from garlic inhibits the NA protein of the influenza A (H1N1) virus, which is responsible for the onset of viral infection.

Garlic-derived DATS treatment applied to human lung epithelial cells reduced viral load, increased viral gene expression, and decreased proinflammatory cytokine expression.

Garlic compounds such as glutamyl cysteine, DATS, allin or allicin possess HIV-1 inhibitory activity.

Immuno-modulatory The consumption of garlic and its derivatives has been shown to exert activity immunomodulatory activity depending on the dose administered.

Garlic's protein fraction and AGE have been shown to stimulate lymphocytes, natural killer cells and macrophages.
References

Liu et al. 1992

Borlinghaus et al. 2014

Askari et al. 2021

Kay et al. 2010

Hiramatsu et al. 2016

Zhang et al. 2017

Liu et al. 2015

Corzo-Martínez et al. 2007

Khanum et al. 2004

Quintero-Fabián et al. 2013

Moutia et al. 2016

Hodge, Hodge, and Han 2002

Larypoor et al. 2013

Liu et al. 2009

Rodrigues \& Percival 2019

Marefati et al. 2020

Fratianni et al. 2016

Nakamoto et al. 2020

Li et al. 2014

Li et al. 2016

Rouf et al. 2020

Pandey et al. 2019

Sen et al. 2020

Thuy et al. 2020

Donma \& Donma 2020

Mehrbod et al. 2009

Sahoo et al. 2016

Ming et al. 2021

Gökalp et al. 2018

Kuttan 2000

Charron et al. 2015 Arreola

et al. 2015

Colić et al. 2002

Ebrahimi et al. 2013

Moutia, Habti, \&

Badou 2018

Rodrigues \& Percival 2019

Percival 2016

SOD: superoxide dismutase; GSH: glutathione; Nrf2: nuclear factor erythroid 2-related factor 2; GCL: glutamate-cysteine ligase; AGE: aged garlic extract; HO-1: heme oxygenase-1; IL: interleukin; MCP-1: monocyte chemotactic protein; TNF- $\alpha$ : tumor necrosis factor $\alpha$; NF-кB: nuclear kappa B transcription factor; NA: neuraminidase; DATS; diallyl trisulfide; HIV-1: human immunodeficiency virus type 1.

Nrf2 pathway. $^{28}$ It was also observed that allicin has a protective effect in vitro against traumatic or ischemic neuronal damage by regulating apoptosis and oxidative stress pathways. $^{29}$
The antioxidant properties of garlic also act by reducing free radicals. Thus, Corzo-Martínez et al. reported that AGE can counter ultraviolet radiation-induced DNA damage. ${ }^{30}$ Antimutagenic activity has been shown by certain sulfur com- 
ponents (e.g., DAS) through their action on DNA repair mechanisms, avoiding the onset of carcinogenesis. ${ }^{31}$

Locatelli et al. ${ }^{6}$ highlighted the antioxidant capacity of allicin, ajoenes, and vinyldithiins (2-VD) in garlic, reporting that allicin can eliminate 1,1-diphenyl-2-picrylhydrazyl radical $\left(\mathrm{DPPH}^{\circ}\right)$ and 2,20-azino-bis-(3-ethylbenzo thiazoline-6-sulfonic acid) diammonium salt (ABTS) and reduce iron levels.

\section{Anti-inflammatory activity}

The anti-inflammatory activity of garlic extracts is well documented. Quintero-Fabián et al. reported that treatment of LPSstimulated 3 T3-L1 adipocytes with alliin reduces the gene expression and protein synthesis of interleukin-6 (IL-6) and monocyte chemotactic protein (MCP-1) and upregulates genes related to the immune response. ${ }^{32}$ Other authors found that treatment with garlic extract inhibited the expression of IL-17 by peripheral blood mononuclear cells without affecting their expression of IL-4. ${ }^{33}$

In another in vitro study, Hodge et al. reported that AGE reduces the production of IL-12 and other proinflammatory cytokines and increases IL-10, which inhibits the production of tumor necrosis factor $\alpha(\mathrm{TNF}-\alpha)$ and IL- $6 .{ }^{34}$ Inhibition of Th1 proinflammatory cytokines by allicin was observed by Larypoor et al..$^{35}$ However, a study in rats found that garlic oil had dual effects on the balance of $\mathrm{T}$ helper cells (Th1-Th2) according to the dose, with low doses obtaining a Th1 response whereas high doses a Th2 response. ${ }^{36}$

Nuclear kappa B transcription factor (NF-kB) is a key regulator of the expression of genes involved in inflammation, including the enzymes nitric oxide synthase (iNOS) and cyclooxygenase-2 (COX-2). NF- $\mathrm{kB}$ overactivation is associated with various chronic inflammatory diseases, and suppression of the NF-KB pathway by anti-inflammatory drugs favors their prevention and treatment. ${ }^{26}$ The NF-kB transcription factor can be inhibited by caffeic acid, SAC, and DATS, among other compounds derived from garlic, thereby inhibiting multiple genes that encode cytokines related to the proinflammatory response (e.g., TNF- $\alpha$, IL-1, IL-6, MCP-1, and IL-12). ${ }^{14}$ In another investigation, a garlic protein of $14 \mathrm{kDa}$ isolated from AGE significantly inhibited the production of NO, prostaglandin E (PGE), TNF- $\alpha$, and IL-1 $\beta$ by LPS-activated macrophages in a dose-dependent manner with no toxic effects. As observed in other studies, the anti-inflammatory effect was largely mediated by the inhibition of iNOS and COX-2 expressions through inactivation of the NF-kB pathway. ${ }^{37}$

\section{Antimicrobial activity}

Ample evidence has also been published on the antimicrobial and antifungal properties of garlic and some of its components. ${ }^{38}$ Fresh garlic extract was found to inhibit the growth of Bacillus cereus, Staphylococcus aureus, Micrococcus spp., Escherichia coli, Klebsiella spp., and Proteus spp., using 1\% chloramphenicol as reference antibiotic. ${ }^{39}$ In addition, Fratianni et al. observed that "Rosato" and "Caposele" garlic varieties exert antimicrobial action against Aspergillus versicolor, Penicillum citrinum, and Penicillium expansum. ${ }^{40}$ The sulfur- ous compounds of garlic can impair biofilm formation by acting as quorum-sensing inhibitors, hampering early bacterial adhesion and the secretion of extracellular polymeric substances and virulence factors. ${ }^{36}$ There is evidence that garlic has antifungal activity against Penicillium funiculosum and Candida albicans. ${ }^{41,42}$ It has also been demonstrated antiviral activity against multiple pathogens. It acts by blocking the entry of viruses into cells, inhibiting viral polymerase-RNA, reverse transcriptase, DNA synthesis, and the transcription of immediate-early gene 1 (IEG1) and downregulating the extracellular signal-regulated kinase (ERK)/mitogen activated protein kinase (MAPK) signaling pathway. ${ }^{43}$ Various researchers have explored the therapeutic potential of garlic against SARS-CoV-2 infection disease. Some authors have observed that garlic and its bioactive compounds may act against this disease by inhibiting target protein $6 \mathrm{LU7}$ of SARS-CoV-2. ${ }^{44-46}$ It has also been proposed that garlic may mitigate the effects of COVID-19 on the immune system by strengthening immune system cells and inhibiting the secretion of proinflammatory cytokines and leptin. ${ }^{47}$ Moreover, some authors point out that garlic and its components may also have antiviral activity against influenza A (H1N1) and avian influenza (H9N2) viruses. ${ }^{48-50}$ Similarly, garlic compounds such as glutamyl cysteine, DATS, allin and allicin have been shown to inhibit the activity of human immunodeficiency virus type 1 (HIV-1) ${ }^{51}$ However, numerous studies indicate that supplementation with garlic or its derivatives could exert an undesirable interaction with prescribed antiretroviral drugs against HIV disease. ${ }^{52,53}$

\section{Immunomodulatory activity}

Garlic can contribute to maintaining immune system homeostasis through its immunomodulatory action on different cell populations, alongside its antiapoptotic, antiparasitic, and anticarcinogenic effects. ${ }^{13}$

Animal experiments showed that garlic consumption can increase the number of immune cells and the total number of bone marrow cells. ${ }^{54}$ A study in humans found that expression of the gene encoding the NFAM1 protein is increased by the addition of $5 \mathrm{~g}$ of raw mashed garlic to one meal every day. NFAM1 is a type I membrane receptor that activates cytokine gene promoters (e.g., IL-13 and TNF- $\alpha$ ) and is involved in B cell development and signaling. ${ }^{55}$

Various garlic derivatives have been found to have a dual effect, exerting either a stimulatory or inhibitory effect on monocytes and lymphocytes according to the dose. ${ }^{13,56,57}$ For instance, concanavalin A-stimulated lymphocyte proliferation is inhibited by high doses of garlic extract but increased by low doses. $^{48}$ The same dual effect has been observed with DATS. ${ }^{57}$

The protein fraction of garlic has demonstrated stimulatory effects on lymphocytes, natural killer (NK) cells, and macrophages. Specifically, it was found to increase the mitogenic activity of human peripheral blood lymphocytes and $\mathrm{CD}^{+} \mathrm{T}$ cells, producing an increase in the delayed hypersensitivity reaction and promoting an effective cellular response. ${ }^{58}$

AGE has also exhibited this immunomodulatory property, activating and increasing the population of NK cells. 
Supplementation with AGE in mice increases the activation of NK and cytotoxic T cells in mice and improves the immune response in humans by augmenting the proliferation of NK and $\mathrm{T} \gamma \delta$ cells. ${ }^{11,13,14,59}$

\section{Therapeutic activity against diseases}

Given its antioxidant, immunomodulatory, and anti-inflammatory properties, researchers have explored the usefulness of garlic in the treatment of various diseases (Table 2).

\section{Infectious processes}

Various authors have studied the action of garlic and its components against infectious processes. Thus, the severity and duration of respiratory tract infections and their symptoms were reduced after the administration of allicin versus placebo. ${ }^{60}$ In a mouse study, garlic oil and DADS were found to have protective effects against respiratory tract inflammation caused by cigarette smoke, reducing the inflammatory cell count and the production of IL-1 $\beta$, IL-6, and TNF- $\alpha{ }^{61}$ Similarly, experimental studies in adults and children showed that daily supplementation with allicin or long-releasing garlic capsules for 12 or 20 weeks respectively, significantly reduced common cold virus infections in adults and acute respiratory infections in children, with no side effects as a result of the administration. ${ }^{62,63}$

In a mouse model of induced polymicrobial sepsis, the administration of methyl sucrose 3-formyl-4-methylpentanoate, isolated from garlic, reduced peritoneal fluid colony-forming units (CFUs) produced by the stimulation of ROS generation, diminished lymphocyte apoptosis in the spleen, and inhibited the production of proinflammatory cytokines (e.g., TNF- $\alpha$, IL-1 $\beta$, and IL-6). ${ }^{64}$

Lee et al. evaluated the growth and immune response of laying chickens fed with a mixture of phytogenic extracts (garlic and cilantro) and probiotics. ${ }^{65}$ They reported an increase in the proliferation of lamina propria leukocytes and in their cytotoxic activity against tumor cells, also observing an improvement in the egg production but not growth of the animals. The inhibition of LPS-induced TNF- $\alpha$ and IFN- $\gamma$ secretion and the reduction in fecal $E$. coli count were similar between chickens fed with this mixture and those treated with antibiotics.

\section{Tumoural processes}

Garlic has demonstrated anticarcinogenic properties against colorectal, breast, liver, and gastric cancers, among others. ${ }^{66-69}$ In 2020, Zhang et al. reported that garlic had therapeutic potential not only to prevent but also to treat cancer, proposing possible mechanisms of action. ${ }^{70}$ The aforementioned beneficial effects of garlic on the immune system would also contribute to controlling tumor processes. ${ }^{11}$ In vitro and in vivo studies have demonstrated the capacity of garlic to inhibit cell division, block carcinogen activation, reduce microbiological growth, scavenge ROS, induce superoxide dismutase, increase proinflammatory cytokine release, and reduce cell prolifer- ation. ${ }^{71}$ These effects have been associated with specific compounds of garlic, especially DADS, DATS, SAC, and SAMC. ${ }^{72}$ Treatment with garlic extract attenuated cell migration and induced death in rat sarcoma cells, and pre-exposure to the extract completely inhibited the growth of implanted tumor cells. $^{73}$ Likewise, treatment with protein fractions extracted from garlic reduced the tumor size in mice with implanted breast tumor cells, increasing $\mathrm{T}$ cell activation and the intratumor infiltration of lymphocytes at all studied doses. ${ }^{56}$

The protein fraction of garlic has been found to modulate NK cell activity in mice, while AGE can increase the number and activity of NK cells in healthy individuals and in patients with advanced cancers. ${ }^{11,14,74,75}$

Cytokines also play an important role in antitumor immunity. Supplementation with allicins of Ehrlich ascites carcinoma (EAC) cells treated with tamoxifen (antiestrogenic drug for breast cancer) increased the expression of cytotoxic damage markers and TNF- $\alpha$ levels. ${ }^{76}$ In another study, AGE significantly increased the apoptosis of acute lymphoblastic leukemia cells without altering the activation of $\mathrm{T}$ cells or the production of IFN- $\gamma$, IL-2, and TNF- $\alpha$, whereas $\mathrm{T}$ cell proliferation and cytokine production were reduced by treatment with cytostatic agents. ${ }^{77}$ In a mouse study, treatment with AGE produced a cytokine release pattern that favored Th1 cell action and antitumor immunity. ${ }^{35}$

Other human randomized clinical trials in have evaluated the effectiveness of garlic against disorders resulting from infection with the bacterium Helicobacter pylori. It was found that combined vitamin and garlic supplementation treatment (extract and oil) for 7.3 years reduced the prevalence of precancerous gastric lesions and reduced the risk of mortality from gastric cancer after a follow-up of 22 years. ${ }^{78,79}$ Indeed, this is in accordance with the findings of Ishikawa et al., who observed an improvement in NK cell activity after 6 months of AGE supplementation in patients with advanced pancreatic, liver and colon cancer. ${ }^{80}$ Similarly, other authors demonstrated that daily supplementation with high-dose AGE $(2.4 \mathrm{~mL})$ could inhibit the progression of colorectal adenoma. ${ }^{81}$ None of these studies found significant adverse effects as a consequence of supplementary therapy.

\section{Activity at vascular level}

Garlic has been attributed with beneficial effects on the cardiovascular system by improving lipid profile, endothelial function, and blood pressure. ${ }^{7,82}$

A meta-analysis published by Silagy and Neil in 1994 confirmed that garlic and its derivatives can reduce levels of triglycerides and cholesterol. ${ }^{83}$ Allicin can also suppress cholesterol biosynthesis by inhibiting the key enzymes squalene-monooxygenase and acetyl-CoA synthetase. ${ }^{84,85}$ In addition, $(1 R, 3 S)-1$ methyl-1,2,3,4-tetrahydro- $\beta$-carboline-3-carboxylic acid obtained from garlic was found to prevent adiposity by regulating the expression of genes involved in the lipid metabolism of adipocytes, downregulating lipogenic genes and upregulating lipolytic genes. ${ }^{86}$ Likewise, experimental studies are in agreement with these findings, observing garlic supplementation to 
Table 2 Summary of the therapeutical activity of garlic

Therapeutic

applications

Infectious

processes

processes

Activity at

vascular level

Prebiotic agent

Therapeutic endocrine and metabolic effects
Mechanism of action

The administration of allicin and garlic oil has been shown to have beneficial effects against respiratory tract infections.

Oral allicin supplementation treatment may prevent common cold virus infection.

Daily administration of $300 \mathrm{mg}$ extended-release garlic capsules for 20 weeks reduced

the morbidity of acute respiratory infections in children aged 10-12 years.

Treatment with garlic extract in a sepsis mouse model showed favourable results against Lee et al. $(2015)^{64}$

the infectious process.

Anti-tumour properties have been found in garlic, mainly in some compounds such as DADS, DATS, SAC, and SAMC.

Treatments with garlic extract and garlic protein fractions reduced the tumour activity of $\mathrm{Hu}$ et al. $(2002)^{73}$

rat sarcoma cells and breast tumour cells.

The protein fraction of garlic and AGE was shown to modulate the activity of natural

killer cells in both healthy populations and individuals with advanced cancer pathologies.

Garlic and its components showed a beneficial effect in the treatment of tumours by modulating cytokine novels, such as TNF- $\alpha$, IFN- $\gamma$, IL-2, as well as the activity exerted by T-cells.

Vitamin supplementation in combination with garlic for 7.3 years showed to reduce the prevalence of precancerous gastric lesions.

Oral vitamin and garlic supplementation treatment administered for 7.3 years was

found to reduce the risk of mortality of gastric cancer after a follow-up of more than 22 years.

Daily supplementation treatment with AGE $(500 \mathrm{mg})$ for 6 months in patients with advanced cancer of the digestive system (pancreatic, colon and liver cancer) showed an improvement of NK cell activity.

A 12-month supplemental treatment with high-dose AGE $\left(2.4 \mathrm{~mL} \mathrm{day}^{-1}\right)$ suppressed the number and size of colorectal adenomas.

Garlic and its constituents may reduce disorders related to the cardiovascular system by reducing triglyceride and cholesterol levels.

The components of garlic may regulate lipid metabolism in adipocytes.

Oral garlic supplementation (400 mg per day) for 12 weeks was shown to significantly reduce blood concentrations of transaminases and cholesterol in patients with nonalcoholic fatty liver disease.

Daily supplementation of $800 \mathrm{mg}$ of garlic in patients with polycystic ovary syndrome reduces plasma levels of total cholesterol, LDL, but not HDL.

Supplementation daily with $900 \mathrm{mg}$ of AGE in men with moderate hypercholesterolemia Adler and Holub $(1997)^{89}$

for 12 weeks significantly reduced serum triglyceride and LDL levels, as well as lowered systolic and diastolic blood pressure.

Supplementation treatment of $9.6 \mathrm{mg}$ allicin for 12 weeks in patients with hypercholesterolemia reduced total, LDL and HDL cholesterol levels.

Garlic supplementation $\left(2 \mathrm{~g} \mathrm{day}^{-1}\right)$ in patients with hyperlipidemia significantly

reduced plasma levels of total and LDL cholesterol, and increased HDL after 60 days.

Similarly, this supplementation therapy reduced systolic blood pressure values, but not dyastolic blood pressure.

Oral black AGE supplementation treatment in patients with mild hypercholesterolemia $\left(6 \mathrm{~g} \mathrm{day}^{-1}\right)$ for 12 weeks did not show a reduction in serum LDL and triglyceride levels, although it did significantly increase HDL levels.

Thiosulfinates act as potent inhibitors of platelet aggregation.

$\begin{array}{ll}\text { Thiosulfinates act as potent inhibitors of platelet aggregation. } & \text { Briggs } \text { et al. }(2000)^{93} \\ \text { Components of garlic have been shown to have antihypertensive properties, with similar } & \text { Dubey et al. }(2017)^{95}\end{array}$

results to drugs commonly used to treat hypertension.

Allicin has a protective effect on blood vessels by increasing endothelial cell proliferation, and decreasing lipopolysaccharide-induced apoptosis and inflammation. Fructane present in garlic may stimulate the proliferation of Bifidobacteria. Supplementation with AGE for 3 months showed a significant increase in intestinal microbiota populations such as Lactobacillus and Clostridia.

Treatments with garlic extracts have demonstrated an improvement in glucose, fructosamine and glycosylated hemoglobin levels, and a weight loss in diabetic rats.

In humans, garlic has been demonstrated to improve components of the metabolic syndrome such as increasing high-density cholesterol and reducing waist circumference, weight, arterial blood pressure and insulin resistance.
Ref.

Yarnell $(2018)^{60}$

Ko et al. $(2018)^{61}$

Josling $(2001)^{63}$

Andrianova et al. $(2003)^{62}$

Miraghajani et al. $(2018)^{72}$

Liu et al. $(2019)^{67}$

Zhang et al. $(2020)^{70}$

Ebrahimi et al. $(2013)^{58}$

Moutia, Habti and Badou (2018) ${ }^{11}$

Rodrigues and Percival $(2019)^{14}$

Ishikawa et al. $(2006)^{74}$

Fallah-Rostami et al. $(2013)^{75}$

Suddek $(2014)^{76}$

Hodge et al. $(2008)^{77}$

Larypoor et al. $(2013)^{35}$

You et al. $(2006)^{78}$

Li et al. $(2019)^{79}$

Ishikawa et al. $(2006)^{80}$

Tanaka et al. $(2006)^{81}$

Gupta and Porter (2001) ${ }^{84}$

Silagy and Neil (1994) ${ }^{83}$

Ried (2016) ${ }^{98}$

Baek et al. $(2019)^{86}$

Sangouni et al. $(2020)^{87}$

Zadhoush et al. $(2021)^{88}$

Kannar et al. $(2001)^{90}$

Zeb et al. $(2018)^{91}$

Jung et al. $(2014)^{92}$

Chaupis-Meza et al. $(2014)^{96}$

Gao et al. $(2020)^{97}$

Zhang et al. $(2017)^{28}$

Zhang et al. $(2013)^{101}$

Ried $(2020)^{102}$

Askari et al. $(2021)^{24}$

Kaur et al. $(2016)^{103}$

Thomson et al. $(2016)^{104}$

Wang et al. $(2017)^{105}$

Sangouni et al. $(2021)^{106}$

Yang et al. $(2016)^{107}$

DADS: diallyl disulfide; DATS: diallyl trisulfide; SAC: $S$-allyl-L-cysteine; SAMC: $S$-allyl-mercaptocysteine; AGE: aged garlic extract; LDL: low-density lipoprotein; HDL: high-density lipoprotein. 
be beneficial in reducing plasma levels of total cholesterol and LDL in patients with nonalcoholic fatty liver disease, ${ }^{87}$ polycystic ovary syndrome, ${ }^{88}$ and hyperlipidemia. ${ }^{89-91}$ In contrast, Jung et al. observed that daily supplementation of $6 \mathrm{~g}$ of black AGE in patients with hypercholesterolemia did not decrease LDL, although they did report an increase in HDL. ${ }^{92}$ No adverse effects were reported in any of the patients.

In relation to cardiovascular disorders, thiosulfinates such as allicin have been reported to act as potent inhibitors of platelet aggregation, thereby contributing to the prevention of cardiac and/or cerebral ischemia. ${ }^{93}$ Allicin derived from garlic has also demonstrated antihypertensive properties, ${ }^{94,95}$ attributed to the action of red blood cells on these polysulfides, which leads to the release of $\mathrm{H}_{2} \mathrm{~S} .{ }^{94} \mathrm{H}_{2} \mathrm{~S}$ is a powerful signaling molecule that relaxes smooth muscle cells surrounding blood vessels and allows their expansion, thereby reducing blood pressures. Chaupis-Meza et al. found that macerated garlic extract had a dose-dependent hypotensive effect in an in vivo rat model, observing a dose-dependent hypotensive effect comparable to that of captopril, widely prescribed for this purpose. ${ }^{96}$ Dubey et al. were not able to identify the underlying mechanism. ${ }^{95}$ However, Gao et al., who verified the anti-hypertensive effects of garlic and its hydrolysates, proposed that they may act by inhibiting the activity of angiotensin-converting enzyme (ACE), reducing the formation of ACE-II, and protecting bradykinin activity. ${ }^{97}$ A meta-analysis of human studies confirmed that garlic contributes to reducing the blood pressure of hypertensive individuals and improving their cholesterol levels. ${ }^{98}$ However, its effect on normotensive individuals has not been established.

Garlic also has a protective effect at endothelial level. Thus, allicin increases the proliferation in vitro of human umbilical vein endothelial cells. ${ }^{28}$ In this regard, the proliferative capacity of this cell population is diminished in response to LPS and the cells release lactate-dehydrogenase, a marker of cytotoxicity. The presence of allicin has been found to decrease LPS-induced apoptosis, suppress ROS overproduction, reduce lipid peroxidation, and decrease endogenous antioxidant enzymatic activities in endothelial cells. ${ }^{99}$ These protective effects have been attributed to the inhibition of mitochondrial dysfunction through a reduction in potential mitochondrial membrane collapse, cytochrome c synthesis, and mitochondrial ATP release. ${ }^{28}$ It has been reported that allicin not only inhibits the LPSinduced inflammatory response but also dose-dependently increases the expression of liver X receptors $\alpha$ (LXR $\alpha)$, and the nuclear receptors LXR $\alpha$ and $\beta$ play a crucial role in regulating carbohydrate and lipid metabolism. ${ }^{28}$ It was also been observed that allicin can activate Nrf2 transcription factor, which regulates defenses against oxidative stress and inflammation (see above). ${ }^{28,100}$ In conclusion, allicin is able to attenuate the LPSinduced inflammatory response at blood vessel level.

\section{Other effects}

Besides the aforementioned benefits, various studies have demonstrated the therapeutic potential of garlic as a prebiotic agent capable of acting on and increasing the diversity of intestinal microbiota. Thus, garlic fructane was found to stimulate the proliferation of Bifidobacteria, ${ }^{101}$ and a meta-analysis by Ried et al. concluded that supplementation with AGE for three months significantly augments populations of Lactobacillus and Clostridia. ${ }^{102}$ Garlic has also shown therapeutic effects at endocrinal and metabolic level. For instance, treatment of type I and II diabetic rats with garlic and certain garlic extracts (e.g., AGE) improved their weight and their levels of glucose, fructosamine, and glycosylated hemoglobin, thereby reducing the damage to pancreatic cells. ${ }^{24,103-105}$ Garlic has also been reported to improve components of the metabolic syndrome in humans by increasing high-density cholesterol and reducing waist circumference, weight, arterial pressure, and insulin resistance. ${ }^{106,107}$

\section{Conclusions}

Garlic and its multiple derivatives possess biological activities with high therapeutic potential against infectious, immunological, vascular, and tumor processes. They can be prepared in raw, macerated, or powder form or as an aqueous, oily, or alcoholic extract. Little clinical advantage has been taken of this natural resource, and further research is warranted in humans to support its therapeutic application.

\section{Author contributions}

Formulation of research question: L. M. R. and C. R.; conception and design of study: L. M. R., R. I. M., C. R. and. V. J. C. R.; literature search and data collection: L. M. R., E. G. R. and C. R.; data analysis: L. M. R., E. G. R., C. R., E. L. B., R. I. M. and V. J. C. R.; writing-original draft preparation, L. M. R., C. R., R. I. M. and V. J. C. R.; writingreview and editing: L. M. R., E. G. R., C. R., E. L. B., R. I. M. and V. J. C. R.; supervision: R. I. M. and C. R. All authors have read and agreed to the published version of the manuscript.

\section{Conflicts of interest}

There are no conflicts to declare.

\section{Acknowledgements}

This study was supported by research group BIO277 (Junta de Andalucía) and Department of Nursing (University of Granada).

\section{Notes and references}

1 E. Ayaz and H. C. Alpsoy, Garlic (Allium sativum) and traditional medicine, Turkiye Parazitol. Derg., 2007, 31, 145-149. 
2 M. Foroutan-Rad, K. H. Tappeh and S. Khademvatan, Antileishmanial and Immunomodulatory Activity of Allium sativum (Garlic): A Review, J. Evidence-Based Complementary Altern. Med., 2017, 22, 141-155.

3 K. Sharma, N. Mahato, S. H. Nile, E. T. Lee and Y. R. Lee, Economical and environmentally-friendly approaches for usage of onion (Allium cepa L.) waste, Food Funct., 2016, 7, 3354-3369.

4 X. Lu, N. Li, X. Qiao, Z. Qiu and P. Liu, Composition analysis and antioxidant properties of black garlic extract, J. Food Drug Anal., 2017, 25, 340-349.

5 K. A. Szychowski, K. Rybczyńska-Tkaczyk, K. Gaweł-Bęben, M. Świeca, M. Karaś, A. Jakubczyk, M. Matysiak, U. E. Binduga and J. Gmiński, Characterization of Active Compounds of Different Garlic (Allium sativum L.) Cultivars, Pol. J. Food Nutr. Sci., 2018, 68, 73-81.

6 D. A. Locatelli, M. A. Nazareno, C. M. Fusari and A. B. Camargo, Cooked garlic and antioxidant activity: Correlation with organosulfur compound composition, Food Chem., 2017, 220, 219-224.

7 G. El-Saber Batiha, A. Magdy Beshbishy, L. G. Wasef, Y. H. A. Elewa, A. A. Al-Sagan, M. E. Abd El-Hack, A. E. Taha, Y. M. Abd-Elhakim and H. Prasad Devkota, Chemical Constituents and Pharmacological Activities of Garlic (Allium sativum L.): A Review, Nutrients, 2020, 12, 872.

8 N. Martins, S. Petropoulos and I. C. F. R. Ferreira, Chemical composition and bioactive compounds of garlic (Allium sativum L.) as affected by pre- and post-harvest conditions: A review, Food Chem., 2016, 211, 41-50.

9 D. Gyawali, R. Vohra, D. Orme-Johnson, S. Ramaratnam and R. H. Schneider, A Systematic Review and MetaAnalysis of Ayurvedic Herbal Preparations for Hypercholesterolemia, Medicina, 2021, 57, 546.

10 M. K. Ang-Lee, J. Moss and C. S. Yuan, Herbal medicines and perioperative care, J. Am. Med. Assoc., 2001, 286, 208216.

11 M. Moutia, N. Habti and A. Badou, In Vitro and In Vivo Immunomodulator Activities of Allium sativum L, J. Evidence-Based Complementary Altern. Med., 2018, 2018, 4984659.

$12 \mathrm{H}$. Amagase, Clarifying the real bioactive constituents of garlic, J. Nutr., 2006, 136, 716S-725S.

13 R. Arreola, S. Quintero-Fabián, R. I. López-Roa, E. O. Flores-Gutiérrez, J. P. Reyes-Grajeda, L. CarreraQuintanar and D. Ortuño-Sahagún, Immunomodulation and anti-inflammatory effects of garlic compounds, J. Immunol. Res., 2015, 2015, 401630.

14 C. Rodrigues and S. S. Percival, Immunomodulatory Effects of Glutathione, Garlic Derivatives, and Hydrogen Sulfide, Nutrients, 2019, 11, 295.

15 A. Shang, S.-Y. Cao, X.-Y. Xu, R.-Y. Gan, G.-Y. Tang, H. Corke, V. Mavumengwana and H.-B. Li, Bioactive Compounds and Biological Functions of Garlic (Allium sativum L.), Foods, 2019, 8, 246.

16 T. Alide, P. Wangila and A. Kiprop, Effect of cooking temperature and time on total phenolic content, total flavonoid content and total in vitro antioxidant activity of garlic, BMC Res. Notes, 2020, 13, 564.

17 J. F. Rinaldi de Alvarenga, P. Quifer-Rada, S. HurtadoBarroso, M. Illan, X. Torrado-Prat and R. M. LamuelaRaventós, Cuisinomics: MS-based untargeted approach reveals chemical modulation by a recipe during home cooking, Food Res. Int., 2020, 138, 109787.

18 L. D. Lawson and Z. J. Wang, Low allicin release from garlic supplements: a major problem due to the sensitivities of alliinase activity, J. Agric. Food Chem., 2001, 49, 2592-2599.15.

19 N. Miękus, K. Marszałek, M. Podlacha, A. Iqbal, C. Puchalski and A. H. Świergiel, Health Benefits of PlantDerived Sulfur Compounds, Glucosinolates, and Organosulfur Compounds, Molecules, 2020, 25, 3804.

20 C. Borek, Dietary antioxidants and human cancer, Integr. Cancer Ther., 2004, 3, 333-341.

21 K. A. Awan, M. S. Butt, F. Ashfaq, H. Munir and H. A. R. Suleria, Prophylactic Potential of Conventional and Supercritical Garlic Extracts to Alleviate Diet Related Malfunctions, Recent Pat. Food, Nutr. Agric., 2019, 10, 34-47.

22 J. Liu, M. W. Albers, T. J. Wandless, S. Luan, D. G. Alberg, P. J. Belshaw, P. Cohen, C. MacKintosh, C. B. Klee and S. L. Schreiber, Inhibition of T cell signaling by immunophilin-ligand complexes correlates with loss of calcineurin phosphatase activity, Biochemistry, 1992, 31, 3896-3901.

23 J. Borlinghaus, F. Albrecht, M. C. H. Gruhlke, I. D. Nwachukwu and A. J. Slusarenko, Allicin: chemistry and biological properties, Molecules, 2014, 19, 1259112618.

24 M. Askari, H. Mozaffari, M. Darooghegi Mofrad, A. Jafari, P. J. Surkan, M. R. Amini and L. Azadbakht, Effects of garlic supplementation on oxidative stress and antioxidative capacity biomarkers: A systematic review and metaanalysis of randomized controlled trials, Phytother. Res., 2021, 35, 3032-3045.

25 H. Y. Kay, J. Won Yang, T. H. Kim, D. Y. Lee, B. Kang, J.-H. Ryu, R. Jeon and S. G. Kim, Ajoene, a stable garlic byproduct, has an antioxidant effect through Nrf2-mediated glutamate-cysteine ligase induction in HepG2 cells and primary hepatocytes, J. Nutr., 2010, 140, 1211-1219.

26 K. Hiramatsu, T. Tsuneyoshi, T. Ogawa and N. Morihara, Aged garlic extract enhances heme oxygenase- 1 and glutamate-cysteine ligase modifier subunit expression via the nuclear factor erythroid 2-related factor 2-antioxidant response element signaling pathway in human endothelial cells, Nutr. Res., 2016, 36, 143-149.

27 E. N. Lee, Y. W. Choi, H. K. Kim, J. K. Park, H. J. Kim, M. J. Kim, H. W. Lee, K.-H. Kim, S. S. Bae, B. S. Kim and $\mathrm{S}$. Yoon, Chloroform extract of aged black garlic attenuates TNF- $\alpha$-induced ROS generation, VCAM-1 expression, $\mathrm{NF}-\kappa \mathrm{B}$ activation and adhesiveness for monocytes in human umbilical vein endothelial cells, Phytother. Res., 2011, 25, 92-100.

28 M. Zhang, H. Pan, Y. Xu, X. Wang, Z. Qiu and L. Jiang, Allicin Decreases Lipopolysaccharide-Induced Oxidative 
Stress and Inflammation in Human Umbilical Vein Endothelial Cells through Suppression of Mitochondrial Dysfunction and Activation of Nrf2, Cell. Physiol. Biochem., 2017, 41, 2255-2267.

29 S.-G. Liu, P.-Y. Ren, G.-Y. Wang, S.-X. Yao and X.-J. He, Allicin protects spinal cord neurons from glutamateinduced oxidative stress through regulating the heat shock protein 70/inducible nitric oxide synthase pathway, Food Funct., 2015, 6, 321-330.

30 M. Corzo-Martínez, N. Corzo and M. Villamiel, Biological properties of onions and garlic, Trends Food Sci. Technol., 2007, 18, 609-625.

31 F. Khanum, K. R. Anilakumar and K. R. Viswanathan, Anticarcinogenic properties of garlic: a review, Crit. Rev. Food Sci. Nutr., 2004, 44, 479-488.

32 S. Quintero-Fabián, D. Ortuño-Sahagún, M. VázquezCarrera and R. I. López-Roa, Alliin, a garlic (Allium sativum) compound, prevents LPS-induced inflammation in 3 T3-L1 adipocytes, Mediators Inflammation, 2013, 2013, 381815.

33 M. Moutia, F. Seghrouchni, O. Abouelazz, A. Elouaddari, A. Al Jahid, A. Elhou, S. Nadifi, J. Jamal Eddine, N. Habti and A. Badou, Allium sativum L. regulates in vitro IL-17 gene expression in human peripheral blood mononuclear cells, BMC Complementary Altern. Med., 2016, 16, 377.

34 G. Hodge, S. Hodge and P. Han, Allium sativum (garlic) suppresses leukocyte inflammatory cytokine production in vitro: potential therapeutic use in the treatment of inflammatory bowel disease, Cytometry, 2002, 48, 209215.

35 M. Larypoor, M. Bayat, M. H. Zuhair, A. Akhavan Sepahy and M. Amanlou, Evaluation of The Number of CD4(+) CD25(+) FoxP3(+) Treg Cells in Normal Mice Exposed to AFB1 and Treated with Aged Garlic Extract, Cell J., 2013, 15, 37-44.

36 C.-T. Liu, H.-M. Su, C.-K. Lii and L.-Y. Sheen, Effect of supplementation with garlic oil on activity of Th1 and Th2 lymphocytes from rats, Planta Med., 2009, 75, 205-210.

37 S. Z. T. Rabe, T. Ghazanfari, Z. Siadat, M. Rastin, S. Z. T. Rabe and M. Mahmoudi, Anti-inflammatory effect of garlic $14 \mathrm{kDa}$ protein on LPS-stimulatedJ774A.1 macrophages, Immunopharmacol. Immunotoxicol., 2015, 37, 158-164.

38 M. Nakamoto, K. Kunimura, J.-I. Suzuki and Y. Kodera, Antimicrobial properties of hydrophobic compounds in garlic: Allicin, vinyldithiin, ajoene and diallyl polysulfides (Review), Exp. Ther. Med., 2020, 19, 1550-1553.

39 N. Marefati, V. Ghorani, F. Shakeri, M. Boskabady, F. Kianian, R. Rezaee and M. H. Boskabady, A review of anti-inflammatory, antioxidant, and immunomodulatory effects of Allium cepa and its main constituents, Pharm. Biol., 2016, 59, 287-302.

40 F. Fratianni, R. Riccardi, P. Spigno, M. N. Ombra, A. Cozzolino, P. Tremonte, R. Coppola and F. Nazzaro, Biochemical Characterization and Antimicrobial and
Antifungal Activity of Two Endemic Varieties of Garlic (Allium sativum L.) of the Campania Region, Southern Italy, J. Med. Food, 2016, 19, 686-691.

41 W.-R. Li, Q.-S. Shi, H.-Q. Dai, Q. Liang, X. Xie, X.-M. Huang, G. Zhao and L.-X. Zhang, Antifungal activity, kinetics and molecular mechanism of action of garlic oil against Candida albicans, Sci. Rep., 2016, 6, 22805.

42 W.-R. Li, Q.-S. Shi, Q. Liang, X.-M. Huang and Y.-B. Chen, Antifungal effect and mechanism of garlic oil on Penicillium funiculosum, Appl. Microbiol. Biotechnol., 2014, 98, 8337-8346.

43 R. Rouf, S. J. Uddin, D. K. Sarker, M. T. Islam, E. S. Ali, J. A. Shilpi, L. Nahar, E. Tiralongo and S. D. Sarker, Antiviral potential of garlic (Allium sativum) and its organosulfur compounds: A systematic update of pre-clinical and clinical data, Trends Food Sci. Technol., 2020, 104, 219-234.

44 P. Pandey, F. Khan, A. Kumar, A. Srivastava and N. K. Jha, Screening of potent inhibitors against 2019 novel coronavirus (Covid-19) from alliumsativum and allium cepa: An in silico approach, Biointerface Res. Appl. Chem., 2021, 11, 7981-7993.

45 D. Sen, P. Debnath, B. Debnath, S. Bhaumik and S. Debnath, J, Identification of potential inhibitors of SARS-CoV-2 main protease and spike receptor from 10 important spices through structure-based virtual screening and molecular dynamic study, Biomol. Struct. Dyn., 2020, 1-22.

46 B. T. Thuy, T. T. A. My, N. T. T. Hai, L. T. Hieu, T. T. Hoa, H. T. P. Loan, N. T. Triet, T. T. V. Anh, P. T. Quy, P. V. Tat, N. V. Hue, D. T. Quang, N. T. Trung, V. T. Tung, L. K. Huynh and N. T. A. Nhung, Investigation into SARS-CoV-2 Resistance of Compounds in Garlic Essential Oil, ACS Omega, 2020, 5, 8312-8320.

47 M. M. Donma and O. Donma, The effects of allium sativum on immunity within the scope of COVID-19 infection, Med. Hypotheses, 2020, 144, 109934.

48 P. Mehrbod, E. Amini and M. Tavassoti-Kheiri, Antiviral activity of garlic extract on Influenza virus, Iran. J. Virol., 2009, 3, 19-23.

49 L. Ming, Z. Li, X. Li, L. Tang and G. He, Antiviral activity of diallyl trisulfide against H9N2 avian influenza virus infection in vitro and in vivo, Virol. J., 2021, 18, 171.

50 M. Sahoo, L. Jena, S. N. Rath and S. Kumar, Identification of Suitable Natural Inhibitor against Influenza A (H1N1) Neuraminidase Protein by Molecular Docking, Genomics Inform., 2016, 14, 96-103.

51 F. Gökalp, The inhibition effect of garlic-derived compounds on human immunodeficiency virus type 1 and saquinavir, J. Biochem. Mol. Toxicol., 2018, 32, e22215.

52 M. A. Jalloh, P. J. Gregory, D. Hein, Z. R. Cochrane and A. Rodriguez, Dietary supplement interactions with antiretrovirals: a systematic review, Int. J. STD AIDS, 2017, 28, 415.

53 C. Bordes, G. Leguelinel-Blache, J.-P. Lavigne, J.-M. Mauboussin, D. Laureillard, H. Faure, I. Rouanet, 
A. Sotto and P. Loubet, Interactions between antiretroviral therapy and complementary and alternative medicine: a narrative review, Clin. Microbiol. Infect., 2020, 26, 1161-1170.

54 G. Kuttan, Immunomodulatory effect of some naturally occuring sulphur-containing compounds, J. Ethnopharmacol., 2000, 72, 93-99.

55 C. S. Charron, H. D. Dawson, G. P. Albaugh, P. M. Solverson, B. T. Vinyard, G. I. Solano-Aguilar, A. Molokin and J. A. Novotny, A Single Meal Containing Raw, Crushed Garlic Influences Expression of Immunityand Cancer-Related Genes in Whole Blood of Humans, J. Nutr., 2015, 145, 2448-2455.

56 Z. H. Feng, G. M. Zhang, T. L. Hao, B. Zhou, H. Zhang and Z. Y. Jiang, Effect of diallyl trisulfide on the activation of $\mathrm{T}$ cell and macrophage-mediated cytotoxicity, J. Tongji Med. Univ. , 1994, 14, 142-147.

57 M. Colić, D. Vucević, V. Kilibarda, N. Radicević and M. Savić, Modulatory effects of garlic extracts on proliferation of T-lymphocytes in vitro stimulated with concanavalin A, Phytomedicine, 2002, 9, 117-124.

58 M. Ebrahimi, Z. Mohammad Hassan, A. Mostafaie, N. Zare Mehrjardi and T. Ghazanfari, Purified Protein Fraction of Garlic Extract Modulates Cellular Immune Response against Breast Transplanted Tumors in BALB/c Mice Model, Cell J., 2013, 15, 65-75.

59 S. S. Percival, Aged Garlic Extract Modifies Human Immunity, J. Nutr., 2016, 146, 433S-436S.

60 E. Yarnell, Herbs for Viral Respiratory Infections, Altern. Complement. Ther., 2018, 24, 35-43.

61 J.-W. Ko, S.-H. Jeong, H.-J. Kwon, N.-R. Shin, Y.-S. Seo, J.-C. Kim, I.-S. Shin and J.-S. Kim, Preventive Effect of Garlic Oil and Its Organosulfur Component DiallylDisulfide on Cigarette Smoke-Induced Airway Inflammation in Mice, Nutrients, 2018, 10, 1659.

62 I. V. Andrianova, I. A. Sobenin, E. V. Sereda, L. I. Borodina and M. I. Studenikin, Effect of long-acting garlic tablets 'allicor' on the incidence of acute respiratory viral infections in children, Ter. Arkh., 2003, 75, 53-56.

63 P. Josling, Preventing the common cold with a garlic supplement: A double-blind, placebo-controlled survey, Adv. Ther., 2001, 18, 189-193.

64 S. K. Lee, Y. J. Park, M. J. Ko, Z. Wang, H. Y. Lee, Y. W. Choi and Y.-S. Bae, A novel natural compound from garlic (Allium sativum L.) with therapeutic effects against experimental polymicrobial sepsis, Biochem. Biophys. Res. Commun., 2015, 464, 774-779.

65 J. S. Lee, M. J. Kim, S. H. Park, S. B. Lee, T. Wang, U. S. Jung, J. Im, E. J. Kim, K. W. Lee and H. G. Lee, Effects of dietary mixture of garlic (Allium sativum), coriander (Coriandrum sativum) and probiotics on immune responses and caecal counts in young laying hens, J. Anim. Physiol. Anim. Nutr., 2017, 101, e122-e132.

66 Z. Li, X. Ying, F. Shan and J. Ji, The association of garlic with Helicobacter pylori infection and gastric cancer risk: A systematic review and meta-analysis, Helicobacter, 2018, 23, e12532.
67 X. Liu, A. Baecker, M. Wu, J.-Y. Zhou, J. Yang, R.-Q. Han, P.-H. Wang, A.-M. Liu, X. Gu, X.-F. Zhang, X.-S. Wang, M. Su, X. Hu, Z. Sun, G. Li, Z.-Y. Jin, S. Y. Jung, L. Mu, N. He, Q.-Y. Lu, L. Li, J.-K. Zhao and Z.-F. Zhang, Raw Garlic Consumption and Risk of Liver Cancer: A Population-Based Case-Control Study in Eastern China, Nutrients, 2019, 11, 2038.

68 X. Zhou, H. Qian, D. Zhang and L. Zeng, Garlic intake and the risk of colorectal cancer: A meta-analysis, Medicine, 2020, 99, e18575.

69 A. Muhammad, M. A. Ibrahim, O. L. Erukainure, I. Malami and A. Adamu, Spices with Breast Cancer Chemopreventive and Therapeutic Potentials: A Functional Foods Based-Review, Anticancer Agents Med. Chem., 2018, 18, 182-194.

70 Y. Zhang, X. Liu, J. Ruan, X. Zhuang, X. Zhang and Z. Li, Phytochemicals of garlic: Promising candidates for cancer therapy, Biomed. Pharmacother., 2020, 123, 109730.

71 A. Pandrangi, Cancer Chemoprevention by Garlic - A Review, Hereditary Genet., 2015, 4, 147.

72 M. Miraghajani, N. Rafie, H. Hajianfar, B. Larijani and L. Azadbakht, Aged Garlic and Cancer: A Systematic Review, Int. J. Prev. Med., 2018, 9, 84.

73 X. Hu, B. N. Cao, G. Hu, J. He, D. Q. Yang and Y. S. Wan, Attenuation of cell migration and induction of cell death by aged garlic extract in rat sarcoma cells, Int. J. Mol. Med., 2002, 9, 641-643.

74 H. Ishikawa, T. Saeki, T. Otani, T. Suzuki, K. Shimozuma, H. Nishino, S. Fukuda and K. Morimoto, Aged garlic extract prevents a decline of NK cell number and activity in patients with advanced cancer, J. Nutr., 2006, 136, 816S-820S.

75 F. Fallah-Rostami, M. A. Tabari, B. Esfandiari, H. Aghajanzadeh and M. Y. Behzadi, Immunomodulatory activity of aged garlic extract against implanted fibrosarcoma tumor in mice, N. Am. J. Med Sci., 2013, 5, 207-212.

76 G. M. Suddek, Allicin enhances chemotherapeutic response and ameliorates tamoxifen-induced liver injury in experimental animals, Pharm. Biol., 2014, 52, 1009-1014.

77 G. Hodge, S. Davis, M. Rice, H. Tapp, B. Saxon and T. Revesz, Garlic compounds selectively kill childhood pre-B acute lymphoblastic leukemia cells in vitro without reducing T-cell function: Potential therapeutic use in the treatment of ALL, Biologics, 2008, 2, 143-149.

78 W. You, L. M. Brown, L. Zhang, J. Li, M. Jin, Y. Chang, J. Ma, K. Pan, W. Liu, Y. Hu, S. Crystal-Mansour, D. Pee, W. J. Blot, J. F. Fraumeni, G. $\mathrm{Xu}$ and M. H. Gail, Randomized double-blind factorial trial of three treatments to reduce the prevalence of precancerous gastric lesions, J. Natl. Cancer Inst., 2006, 98, 974-983.

79 W.-Q. Li, J.-Y. Zhang, J.-L. Ma, Z.-X. Li, L. Zhang, Y. Zhang, Y. Guo, T. Zhou, J.-Y. Li, L. Shen, W.-D. Liu, Z.-X. Han, W. J. Blot, M. H. Gail, K.-F. Pan and W.-C. You, Effects of Helicobacter pylori treatment and vitamin and garlic supplementation on gastric cancer incidence and mortality: follow-up of a randomized intervention trial, Br. Med. J., 2019, 366, 15016. 
80 H. Ishikawa, T. Saeki, T. Otani, T. Suzuki, K. Shimozuma, H. Nishino, S. Fukuda and K. Morimoto, Aged garlic extract prevents a decline of NK cell number and activity in patients with advanced cancer, J. Nutr., 2006, 136, 816S-820S.

81 S. Tanaka, K. Haruma, M. Yoshihara, G. Kajiyama, K. Kira, H. Amagase and K. Chayama, Aged garlic extract has potential suppressive effect on colorectal adenomas in humans, J. Nutr., 2006, 136, 821S-826S.

82 H. R. Ramírez-Concepción, L. N. Castro-Velasco and E. Martínez-Santiago, Efectos Terapéuticos del Ajo (Allium Sativum), Salud Adm, 2016, 3, 39-47.

83 C. Silagy and A. Neil, Garlic as a lipid lowering agent-a meta-analysis, J. R. Coll. Physicians Lond., 1994, 28, 39-45.

84 N. Gupta and T. D. Porter, Garlic and garlic-derived compounds inhibit human squalene monooxygenase, J. Nutr., 2001, 131, 1662-1667.

85 M. Focke, A. Feld and K. Lichtenthaler, Allicin, a naturally occurring antibiotic from garlic, specifically inhibits acetyl-CoA synthetase, FEBS Lett., 1990, 261, 106-108.

86 S. C. Baek, K. H. Nam, S. A. Yi, M. S. Jo, K. H. Lee, Y. H. Lee, J. Lee and K. H. Kim, Anti-adipogenic Effect of $\beta$-Carboline Alkaloids from Garlic (Allium sativum), Foods, 2019, 8, 673.

87 A. A. Sangouni, M. R. Mohammad Hosseini Azar and M. Alizadeh, Effect of garlic powder supplementation on hepatic steatosis, liver enzymes and lipid profile in patients with non-alcoholic fatty liver disease: a doubleblind randomised controlled clinical trial, Br. J. Nutr., 2020, 124, 450-456.

88 R. Zadhoush, A. Alavi-Naeini, A. Feizi, E. Naghshineh and M. R. Ghazvini, The effect of garlic (Allium sativum) supplementation on the lipid parameters and blood pressure levels in women with polycystic ovary syndrome: A randomized controlled trial, Phytother. Res., 2021, 35, 6335-6342.

89 A. J. Adler and B. J. Holub, Effect of garlic and fish-oil supplementation on serum lipid and lipoprotein concentrations in hypercholesterolemic men, Am. J. Clin. Nutr., 1997, 65, 445-450.

90 D. Kannar, N. Wattanapenpaiboon, G. S. Savige and M. L. Wahlqvist, Hypocholesterolemic effect of an entericcoated garlic supplement, J. Am. Coll. Nutr., 2001, 20, 225231.

91 F. Zeb, M. Safdar, S. Fatima, S. Khan, S. Alam, M. Muhammad, A. Syed, F. Habib and H. Shakoor, Supplementation of garlic and coriander seed powder: Impact on body mass index, lipid profile and blood pressure of hyperlipidemic patients, Pak. J. Pharm. Sci., 2018, 31, 1935-1941.

92 E.-S. Jung, S.-H. Park, E.-K. Choi, B.-H. Ryu, B.-H. Park, D.-S. Kim, Y.-G. Kim and S.-W. Chae, Reduction of blood lipid parameters by a 12-wk supplementation of aged black garlic: a randomized controlled trial, Nutrition, 2014, 30, 1034-1039.

93 W. H. Briggs, H. Xiao, K. L. Parkin, C. Shen and I. L. Goldman, Differential inhibition of human platelet aggregation by selected Allium thiosulfinates, J. Agric. Food Chem., 2000, 48, 5731-5735.

94 G. A. Benavides, G. L. Squadrito, R. W. Mills, H. D. Patel, T. S. Isbell, R. P. Patel, V. M. Darley-Usmar, J. E. Doeller and D. W. Kraus, Hydrogen sulfide mediates the vasoactivity of garlic, Proc. Natl. Acad. Sci. U. S. A., 2007, 104, 17977-17982.

95 H. Dubey, A. Singh, A. M. Patole and C. R. Tenpe, Antihypertensive effect of allicin in dexamethasoneinduced hypertensive rats, Integr. Med. Res., 2017, 6, 6065.

96 D. Chaupis-Meza, J. Rojas, M. Gasco and G. F. Gonzales, Hypotensive effect of extract of macerated garlic (Allium sativum) for 18 weeks in an in vivo experimental model, Rev. Peru Med. Exp. Salud Publica, 2014, 31, 461-466.

97 X. Gao, Z. Xue, Q. Ma, Q. Guo, L. Xing, R. K. Santhanam, M. Zhang and H. Chen, Antioxidant and antihypertensive effects of garlic protein and its hydrolysates and the related mechanism, J. Food Biochem., 2020, 44, e13126.

98 K. Ried, Garlic Lowers Blood Pressure in Hypertensive Individuals, Regulates Serum Cholesterol, and Stimulates Immunity: An Updated Meta-analysis and Review, J. Nutr., 2016, 146, 389S-396S.

99 X. Chen, S. Pang, J. Lin, J. Xia and Y. Wang, Allicin prevents oxidized low-density lipoprotein-induced endothelial cell injury by inhibiting apoptosis and oxidative stress pathway, BMC Complementary Altern. Med., 2016, 16, 133.

100 C.-L. Li, X.-H. Liu, Y. Qiao, L.-N. Ning, W.-J. Li, Y.-S. Sun, D.-S. Liu, W. Gao and C.-M. Ma, Allicin alleviates inflammation of diabetic macroangiopathy via the Nrf2 and NFkB pathway, Eur. J. Pharmacol., 2020, 876, 173052.

101 N. Zhang, X. Huang, Y. Zeng, X. Wu and X. Peng, Study on prebiotic effectiveness of neutral garlic fructan in vitro, Food Sci. Hum., 2013, 2, 119-123.

102 K. Ried, Garlic lowers blood pressure in hypertensive subjects, improves arterial stiffness and gut microbiota: A review and meta-analysis, Exp. Ther. Med., 2020, 19, 14721478.

103 G. Kaur, R. Padiya, R. Adela, U. K. Putcha, G. S. Reddy, B. R. Reddy, K. P. Kumar, S. Chakravarty and S. K. Banerjee, Garlic and Resveratrol Attenuate Diabetic Complications, Loss of $\beta$-Cells, Pancreatic and Hepatic Oxidative Stress in Streptozotocin-Induced Diabetic Rats, Front. Pharmacol., 2016, 7, 360.

104 M. Thomson, K. K. Al-Qattan, D. Js and M. Ali, Anti-diabetic and anti-oxidant potential of aged garlic extract (AGE) in streptozotocin-induced diabetic rats, $B M C$ Complementary Altern. Med., 2016, 16, 17.

105 J. Wang, X. Zhang, H. Lan and W. Wang, Effect of garlic supplement in the management of type 2 diabetes mellitus (T2DM): a meta-analysis of randomized controlled trials, Food Nutr. Res., 2017, 61, 1377571.

106 A. A. Sangouni, M. Alizadeh, A. Jamalzehi and K. Parastouei, Effects of garlic powder supplementation 
on metabolic syndrome components, insulin resistance, fatty liver index, and appetite in subjects with metabolic syndrome: A randomized clinical trial, Phytother. Res., 2021, 35, 4433-4441.
107 D. J. Yang, S. H. Moh, D. H. Son, S. You, A. W. Kinyua, C. M. Ko, M. Song, J. Yeo, Y.-H. Choi and K. W. Kim, Gallic Acid Promotes Wound Healing in Normal and Hyperglucidic Conditions, Molecules, 2016, 21, 899. 